PROCEEDINGS OF THE

AMERICAN MATHEMATICAL SOCIETY

Volume 129, Number 12, Pages 3691-3699

S 0002-9939(01)06167-6

Article electronically published on May 10, 2001

\title{
POLYNOMIAL GROWTH SOLUTIONS OF UNIFORMLY ELLIPTIC OPERATORS OF NON-DIVERGENCE FORM
}

\author{
PETER LI AND JIAPING WANG \\ (Communicated by Bennett Chow)
}

\begin{abstract}
We give an explicit description of polynomial growth solutions to a uniformly elliptic operator of non-divergence form with periodic coefficients on the Euclidean spaces. We also show that the solutions are of one-to-one correspondence to harmonic polynomials if the coefficients of the operator are continuous.
\end{abstract}

\section{$\S 0$. INTRODUCTION}

In this article, we will study polynomial growth solutions to a uniformly elliptic operator of non-divergence form defined on $\mathbb{R}^{n}$. In terms of rectangular coordinates $\left\{x_{1}, \ldots, x_{n}\right\}$ of $\mathbb{R}^{n}$, let

$$
L=a_{i j}(x) \frac{\partial^{2}}{\partial x_{i} \partial x_{j}}
$$

be second order differential operator. We assume that it is symmetric and uniformly elliptic with the coefficients satisfying

$$
a_{i j}(x)=a_{j i}(x)
$$

and

$$
\lambda|\xi|^{2} \leq \sum_{i, j=1}^{n} a_{i j}(x) \xi_{i} \xi_{j} \leq \mu|\xi|^{2}
$$

for some positive constants $0<\lambda \leq \mu<\infty$. Moreover, we assume that the functions $a_{i j}$ are measurable and periodic with respect to the lattice $\mathbb{Z}^{n}$ satisfying

$$
a_{i j}(x+z)=a_{i j}(x)
$$

for all $x \in \mathbb{R}^{n}$ and $z \in \mathbb{Z}^{n}$. We say that a function $u \in W_{l o c}^{2, n}\left(\mathbb{R}^{n}\right)$ is $L$-harmonic if it is a weak solution of the equation

$$
L u=0 .
$$

Received by the editors May 2, 2000.

2000 Mathematics Subject Classification. Primary 35J15.

The first author's research was partially supported by NSF grant \#DMS-9971418.

The second author's research was partially supported by NSF grant \#DMS-9704482. 
Let $r(x)$ be the Euclidean distance function to the origin on $\mathbb{R}^{n}$. For any $d \geq 0$, let us define

$$
\mathcal{H}^{d}(L)=\left\{u \in W_{l o c}^{2, n}\left(\mathbb{R}^{n}\right)|L u=0,| u \mid(x)=O\left(r^{d}(x)\right)\right\}
$$

to be the space of $L$-harmonic functions of polynomial growth of order at most $d$. Our main purpose is to prove the following theorem.

Theorem. If $L$ is a $\mathbb{Z}^{n}$-periodic, uniformly elliptic operator of non-divergence form, then the following properties hold:

(i) $\mathcal{H}^{d}(L)=\mathcal{H}^{[d]}(L)$.

(ii) For any positive integer $N$ and $u \in \mathcal{H}^{N}(L)$,

$$
u(x)=\sum_{|\nu| \leq N} \frac{p_{\nu}(x)}{\nu !} x^{\nu},
$$

where $\nu=\left(\nu_{1}, \ldots, \nu_{n}\right) \in \mathbb{Z}_{+}^{n},|\nu|=\sum_{i=1}^{n} \nu_{i}, \nu !=\prod_{i=1}^{n} \nu_{i} !$ and $x^{\nu}=\prod_{i=1}^{n} x_{i}^{\nu_{i}}$. The functions $p_{\nu}(x)$ are $\mathbb{Z}^{n}$-periodic and Hölder continuous. Moreover, when $|\nu|=N$ the functions $p_{\nu}(x)$ are constants.

(iii) If $a_{i j}$ are continuous, then the homogeneous polynomial

$$
u^{(N)}(x)=\sum_{|\nu|=N} \frac{p_{\nu}(x)}{\nu !} x^{\nu}
$$

solves

$$
Q u^{(N)}=\sum_{i, j=1}^{n} q_{i j} \frac{\partial^{2} u^{(N)}}{\partial x_{i} \partial x_{j}}=0,
$$

where $\left(q_{i j}\right)$ is a positive definite constant matrix. In particular,

$$
\operatorname{dim} \mathcal{H}^{N}(L)=\operatorname{dim} \mathcal{H}^{N}(\Delta) .
$$

Let us first point out some history relating to this particular problem. In 1989 ALn, Avenalleda and Lin studied solutions to a uniformly elliptic operator of divergence form on $\mathbb{R}^{n}$. In their setting, they consider elliptic operators of the form

$$
P=\frac{\partial}{\partial x_{i}}\left(a_{i j}(x) \frac{\partial}{\partial x_{j}}\right)
$$

defined on $\mathbb{R}^{n}$. They assumed that the coefficients $\left\{a_{i j}(x)\right\}$ of the operator are Lipschitz functions, satisfying (0.2), (0.3) and period under the $\mathbb{Z}^{n}$ action. They proved that the dimension of the space of polynomial growth solutions of degree at most $d, \mathcal{H}^{d}(P)$, must satisfy

$$
\operatorname{dim} \mathcal{H}^{d}(P)=\operatorname{dim} \mathcal{H}^{d}(\Delta),
$$

where $\Delta$ is the Laplace operator on $\mathbb{R}^{n}$. Three years later, Moser and Struwe MS] used a different argument and proved the same theorem without the Lipschitz assumption on the coefficients. Moreover, their argument applied to a certain class of non-linear operators as well.

In 1996, Lin [Ln] considered uniformly elliptic operators of divergence form which are asymptotic to a conic operator at infinity. This result can be viewed as a generalization of the previous results since a periodic operator is asymptotic to an operator with constant coefficients. Recently, Zhang [Z] considered a more general class, namely operators that are asymptotic to a compact 1-parameter family of 
conic operators. In this setting, he only proved an upper bound on the dimension of $\mathcal{H}^{d}(P)$.

The most general theorem on uniformly elliptic operators of divergence form was included in the work [L1] of the first author. He showed that for any uniformly elliptic operator of divergence form with measurable coefficients the dimension of $\mathcal{H}^{d}(P)$ must satisfy

$$
\operatorname{dim} \mathcal{H}^{d}(P) \leq C d^{n-1}
$$

for all $d \geq 1$. In fact, his argument also works for uniformly elliptic operators, $L$, of non-divergence form (0.1) with measurable coefficients satisfying (0.2) and (0.3). In that case, the upper bound

$$
\operatorname{dim} \mathcal{H}^{d}(L) \leq C d^{n-1}
$$

is obtained.

In a recent paper $[\mathrm{LW}]$, the authors found a sharp asymptotic upper bound for the dimension of $\mathcal{H}^{d}(P)$ as $d \rightarrow \infty$, for uniformly elliptic operators of divergence form with measurable coefficients. They showed that

$$
\sum_{i=1}^{d} \operatorname{dim} \mathcal{H}_{i}(P) \leq\left(\frac{\mu_{\infty}}{\lambda_{\infty}}\right)^{n-1} \frac{2}{n !}(d+2 n)^{n}
$$

where $\lambda_{\infty}$ and $\mu_{\infty}$ are the ellipticity constants at infinity. This estimate is sharp in the sense that for the Laplace operator, we have

$$
\lim _{d \rightarrow \infty} d^{-n} \sum_{i=1}^{d} \operatorname{dim} \mathcal{H}_{i}(\Delta)=\frac{2}{n !} .
$$

An obvious question is to ask if the above theories apply to uniformly elliptic operators, $L$, of non-divergence form. Other than the result in [L1], there are no known results for this class of operators. The first step in our attempt to obtain some form of sharp estimates on $\operatorname{dim} \mathcal{H}^{d}(L)$ is to see if similar results such as ALn and [MS] are valid. Our program follows pretty much that of Moser-Struwe with the exception that different treatments are used in various crucial steps to deal with the non-divergence case.

We would like to remark that the continuity assumption of the coefficients is only required in discussing the solvability of the equation

$$
L u=f .
$$

It is likely that the theorem also holds for measurable coefficients. Finally, we would like to refer the interested reader to a comprehensive survey [L2] by the first author on harmonic functions.

We would like to thank P. Kuchment and Y. Pinchover for informing us about their recent paper $[\mathrm{KP}$, in which they have used Floquet theory to obtain many interesting results concerning the representations of solutions to general periodic elliptic equations with smooth coefficients. Consequently, they were able to conclude the similar results proven here. In fact, they have also outlined an argument similar to ours in the appendix of their paper. In any case, it is still unclear to us how to deal with the measurable coefficient case. 


\section{§1. Proof of the Theorem}

We will now give a proof of the Theorem.

Proof. Denote $E_{i}$ to be the translation operator defined on functions given by

$$
\left(E_{i} u\right)(x)=u\left(x+e_{i}\right)-u(x),
$$

where $e_{i}=(0, \ldots, 1, \ldots, 0)$ is the $i$-th Cartesian coordinate vector. We also denote $E^{\nu}=\prod_{i=1}^{n} E_{i}^{\nu_{i}}$ for $\nu=\left(\nu_{1}, \ldots, \nu_{n}\right) \in \mathbb{Z}_{+}^{n}$.

As a general fact, we will first show that

$$
u(x)=\sum_{|\nu|<N} p_{\nu}(x) x^{\nu}
$$

if $E^{\nu} u=0$ for all $\nu$ with $|\nu|=N$, where each $p_{\nu}(x)$ is periodic. We will prove this by induction on $N$. For $N=1$, if $E_{i} u=0$ for all $i=1, \ldots, n$, then $u$ is $\mathbb{Z}^{n}$-periodic and the result is trivially true. Suppose now that this is true for $N=k$ and we would like to prove it for $N=k+1$. Note that for any $\nu \in \mathbb{Z}_{+}^{n}$ with $|\nu|=k$, the assumption asserts that $E_{i}\left(E^{\nu} u\right)=0$ for all $i=1, \ldots, n$. This implies that $E^{\nu} u=p_{\nu}(x)$ for some periodic function $p_{\nu}$. Now for a fixed $\nu_{0} \in \mathbb{Z}_{+}^{n}$ with $\left|\nu_{0}\right|=k$, one checks directly that

$$
E^{\nu_{0}}\left(u(x)-\sum_{|\nu|=k} \frac{p_{\nu}(x)}{\nu !} x^{\nu}\right)=E^{\nu_{0}} u-p_{\nu_{0}}(x)=0 .
$$

Hence the induction hypothesis implies that

$$
u(x)-\sum_{|\nu|=k} \frac{p_{\nu}(x)}{\nu !} x^{\nu}=\sum_{|\nu|<k} \frac{p_{\nu}(x)}{\nu !} x^{\nu} .
$$

Rewrite this as

$$
u(x)=\sum_{|\nu| \leq k} \frac{p_{\nu}(x)}{\nu !} x^{\nu}
$$

the induction argument is complete.

To continue with the proof of the theorem, let us denote $\omega_{B_{x}(R)}(f)$ to be the oscillation of a function over the ball $B_{x}(R)$. The Harnack inequality of KrylovSafanov [KS1, KS2] implies that there exists a positive constant $C<1$ depending only on $\lambda, \mu$ and $n$, such that, if $f \in W_{l o c}^{2, n}\left(\mathbb{R}^{n}\right)$ is an $L$-harmonic function, then

$$
\omega_{B_{x}(R)}(f) \leq C \omega_{B_{x}(2 R)}(f)
$$

for all $x \in \mathbb{R}^{n}$ and $R>0$. We claim that for any $u \in \mathcal{H}^{d}(L), E_{i} u \in \mathcal{H}^{d-\delta}(L)$ for all $i=1, \ldots, n$, where $\delta=\log _{2} \frac{1}{C}>0$. In fact, it is clear that if we define

$$
\begin{aligned}
v(x) & =E_{i} u(x) \\
& =u\left(x+e_{i}\right)-u(x),
\end{aligned}
$$


then $L v=0$ and $v \in W_{l o c}^{2, n}\left(\mathbb{R}^{n}\right)$. For any $x \in \mathbb{R}^{n}$, let $k$ be the minimum positive integer such that $r(x) \leq 2^{k}$. Using (1.1), we get

$$
\begin{aligned}
|v|(x) & \leq \omega_{B_{x}(1)}(u) \\
& \leq C^{k} \omega_{B_{x}\left(2^{k}\right)}(u) \\
& \leq C^{k} A\left(r(x)+2^{k}\right)^{d} \\
& \leq B r^{d-\delta}(x) .
\end{aligned}
$$

This implies that $v \in \mathcal{H}^{d-\delta}(L)$ and the claim follows.

A standard argument using the Harnack inequality also implies that there exists $\alpha>0$ such that $\mathcal{H}^{\alpha}(L)$ only consists of constant functions. For a fixed $d>0$, there exists a positive integer $m$ such that $d-m \delta \leq \alpha$. Therefore if $u \in \mathcal{H}^{d}(L)$ and $\nu \in \mathbb{Z}_{+}^{n}$ with $|\nu|=m$, then $E^{\nu} u \in \mathcal{H}^{d-m \delta}(L)$ must be a constant. In particular, for $\nu \in \mathbb{Z}_{+}^{n}$ with $|\nu|=m+1, E^{\nu} u=0$. Hence we conclude that

$$
u(x)=\sum_{|\nu| \leq m} \frac{p_{\nu}(x)}{\nu !} x^{\nu},
$$

where the functions $p_{\nu}(x)$ are periodic. However, the growth order of $u$ is of at most $d$. The non-zero terms in (1.2) must satisfy $|\nu| \leq[d]$. Thus, $\mathcal{H}^{d}(L)=\mathcal{H}^{[d]}(L)$ and (i) is proved.

Using (1.2), we conclude that if $u \in \mathcal{H}^{N}(L)$ with $N$ being a positive integer, then

$$
u(x)=\sum_{|\nu| \leq N} \frac{p_{\nu}(x)}{\nu !} x^{\nu} .
$$

Direct computation then shows that $E^{\nu} u=p_{\nu}(x) \in \mathcal{H}^{0}(L)$ for $|\nu|=N$. Due to the fact that the space $\mathcal{H}^{0}(L)$ consists of constant functions only, we conclude that $p_{\nu}$ must be constant for $|\nu|=N$. The Hölder regularity of $L$-harmonic functions implies that all the $p_{\nu}(x)$ are Hölder continuous.

To show (iii), we first remark that we may view $L$ as an operator on the torus $T^{n}$. The standard $L^{p}$ estimate implies that the image of $H^{2}\left(T^{n}\right)$ under $L$ is a closed subspace of $L^{2}\left(T^{n}\right)$. Note that by the maximum principle, positive functions are not in the image space. This implies that the kernel of the dual operator $L^{*}: L^{2}\left(T^{n}\right) \rightarrow H^{2}\left(T^{n}\right)$ is non-trivial. Let $f \in L^{2}\left(T^{n}\right)$ be a non-zero function in the kernel of $L^{*}$. Without loss of generality, we may assume that

$$
\int_{T^{n}} f \geq 0
$$

We now claim that $f$ must be non-negative on $T^{n}$. In fact, for any non-negative smooth function $h$ on $T^{n}$, let $w(x, t)$ be the solution of

$$
\left(L-\frac{\partial}{\partial t}\right) w=0
$$

with

$$
w(x, 0)=h(x) .
$$

Note that by the $L^{p}$ estimate, $w(x, t) \in H^{2}\left(T^{n}\right)$ for all $t>0$ and

$$
\lim _{t \rightarrow \infty} w(x, t)=c
$$


for some constant $c \geq 0$. Since $L^{*}(f)=0$ and $w(x, t) \in H^{2}\left(T^{n}\right)$ for $t>0$,

$$
\int_{T^{n}} f(x) L w(x, t) d x=0
$$

for all $t>0$. Thus,

$$
\frac{d}{d t} \int_{T^{n}} f(x) w(x, t) d x=0
$$

and

$$
\begin{aligned}
\int_{T^{n}} f(x) h(x) d x & =\lim _{t \rightarrow \infty} \int_{T^{n}} f(x) w(x, t) d x \\
& =c \int_{T^{n}} f(x) d x \\
& \geq 0 .
\end{aligned}
$$

Since $h$ is arbitrary, we conclude that $f(x) \geq 0$ on $T^{n}$. Let us now normalize $f$ such that

$$
\int_{T^{n}} f(x) d x=1
$$

and define

$$
q_{i j}=\int_{T^{n}} a_{i j}(x) f(x) d x .
$$

Since $f \geq 0$ and is not identically zero, $q_{i j}$ is positive definite. We will now verify that $Q u^{(N)}=0$ with this choice of $q_{i j}$. This is obvious for $N=1$ as $u^{(1)}(x)=c_{i} x_{i}$ for some constants $c_{i}$. For $N=2$ and $u \in \mathcal{H}^{2}(L)$, (ii) implies that there exists a periodic function $p$ such that

$$
u(x)=c_{i j} x_{i} x_{j}+c_{i} x_{i}+p(x) .
$$

Hence $u^{(2)}(x)=c_{i j} x_{i} x_{j}$, where $c_{i j}$ are constants. Therefore, we have

$$
\begin{aligned}
Q\left(u^{(2)}\right) & =q_{i j} c_{i j} \\
& =\int_{T^{n}} f(x) a_{i j}(x) c_{i j} d x \\
& =\int_{T^{n}} f(x) L\left(u^{(2)}\right)(x) d x \\
& =-\int_{T^{n}} f(x) L(p)(x) d x \\
& =-\int_{T^{n}} L^{*}(f)(x) p(x) d x \\
& =0 .
\end{aligned}
$$

We will now proceed by induction for $N \geq 3$. Suppose for all $u \in \mathcal{H}^{(N-1)}(L)$ we have $Q u^{(N-1)}=0$. Given $u \in \mathcal{H}^{N}(L)$, we know that $E_{i} u \in \mathcal{H}^{(N-1)}(L)$ for each $i$. Note that

$$
\frac{\partial u^{(N)}}{\partial x_{i}}=\left(E_{i} u\right)^{(N-1)},
$$


so by the induction hypothesis, we have

$$
\begin{aligned}
\frac{\partial\left(Q u^{(N)}\right)(x)}{\partial x_{i}} & =Q\left(\frac{\partial u^{(N)}}{\partial x_{i}}\right) \\
& =0 .
\end{aligned}
$$

This implies that the function $Q u^{(N)}$ is constant. On the other hand, $Q u^{(N)}$ is a homogeneous polynomial of degree $N-2>0$, hence it is identically zero. This implies the first part of (iii).

To prove the second part of (iii), note that the correspondence of $u \mapsto u^{(N)}$ gives a one-to-one map from $\mathcal{H}^{N}(L) / \mathcal{H}^{(N-1)}(L)$ to $\mathcal{H}^{N}(Q) \mathcal{H}^{(N-1)}(Q)$. We will now show that this map is onto, that is, for any $h(x)=\sum_{|\nu|=N} c_{\nu} x^{\nu} \in \mathcal{H}^{N}(Q)$, there exists $u \in \mathcal{H}^{N}(L)$ such that $u^{(N)}=h$. Let us write

$$
u(x)=\sum_{|\nu| \leq N} p_{\nu}(x) x^{\nu}
$$

where $p_{\nu}(x)=c_{\nu}$ for $|\nu|=N$. For $\epsilon>0$, we have

$$
\begin{aligned}
\epsilon^{N} u\left(\frac{x}{\epsilon}\right) & =\sum_{|\nu| \leq N} \epsilon^{N-|\nu|} x^{\nu} p_{\nu}\left(\frac{x}{\epsilon}\right) \\
& =U(x, y, \epsilon),
\end{aligned}
$$

after substituting the new variable $y=\frac{x}{\epsilon}$. Direct calculation shows that $L u=0$ if and only if

$$
\left(L_{0}+\epsilon L_{1}+\epsilon^{2} L_{2}\right) U(x, y, \epsilon)=0
$$

where

$$
\begin{gathered}
L_{0}=a_{i j}(y) \frac{\partial^{2}}{\partial y_{i} \partial y_{j}}, \\
L_{1}=a_{i j}(y)\left(\frac{\partial^{2}}{\partial x_{i} \partial y_{j}}+\frac{\partial^{2}}{\partial y_{i} \partial x_{j}}\right)
\end{gathered}
$$

and

$$
L_{2}=a_{i j}(y) \frac{\partial^{2}}{\partial x_{i} \partial x_{j}}
$$

We now claim that there exists a sequence of functions $\psi_{\nu}(y)$ such that $\psi_{0}(y)=1$ and

$$
\begin{aligned}
&\left(L_{0}+\epsilon\right.\left.L_{1}+\epsilon^{2} L_{2}\right) \sum_{|\nu|=0}^{\infty} \epsilon^{|\nu|} \psi_{\nu}(y) \frac{\partial^{|\nu|}}{\partial x^{\nu}} \\
&=\epsilon^{2} \sum_{|\nu|=2} \alpha_{\nu} \frac{\partial^{|\nu|}}{\partial x^{\nu}}+\epsilon^{3} \sum_{|\nu|=3} \alpha_{\nu} \frac{\partial^{|\nu|}}{\partial x^{\nu}}+\ldots
\end{aligned}
$$

where $\alpha_{\nu}$ are constants and $\alpha_{i j}=q_{i j}$. In fact, comparing the coefficients of $\epsilon^{s}$ and the corresponding terms of $\frac{\partial^{|\nu|}}{\partial x^{\nu}}$ of (1.4), we get

$$
L_{0} \psi_{0}(y)=0 \quad \text { for } s=0
$$

and

$$
L_{0} \psi_{\nu}(y)=0 \quad \text { for }|\nu|=s=1
$$


Let us set $\psi_{\nu}(y)=0$ for all $\nu$ with $|\nu|=1$. For $s=2$, we have

$$
L_{0} \psi_{i j}(y)+a_{i j}(y)=\alpha_{i j}
$$

for all $i, j=1, \ldots, n$. Setting $\alpha_{i j}=q_{i j}$ and using the normalization on $f$, we check that

$$
\int_{T^{n}} f(y)\left(\alpha_{i j}-a_{i j}(y)\right) d y=0 .
$$

Hence there exist solutions $\psi_{i j}(y)$ to (1.5). We will now use an induction argument on $s \geq 3$. Let us assume that $\psi_{\nu}(y)$ exists for all $\nu$ with $|\nu| \leq s-1$. Using (1.5), we know that for each $\nu$ with $|\nu|=s$,

$$
L_{0} \psi_{\nu}(y)=F\left(\psi_{\nu_{1}}, \psi_{\nu_{2}}\right)(y)+\alpha_{\nu},
$$

where $\left|\nu_{1}\right|=s-1$ and $\left|\nu_{2}\right|=s-2$. So we may choose $\alpha_{\nu}$ such that the right-hand side of (1.6) is perpendicular to $f(y)$ in $L^{2}\left(T^{n}\right)$. Thus, solutions $\psi_{\nu}$ exist for (1.6) and the claim is proved.

Note that the operator $Q$ maps the set of homogeneous polynomials of degree $s$ onto the set of homogeneous polynomials of degree $s-2$. There exists a linear operator $R$ mapping polynomials to polynomials such that $Q R=I$. Let us define formally

$$
A=I+\epsilon R \sum_{|\nu|=3} \alpha_{\nu} \frac{\partial^{|\nu|}}{\partial x^{\nu}}+\epsilon^{2} R \sum_{|\nu|=4} \alpha_{\nu} \frac{\partial^{|\nu|}}{\partial x^{\nu}}+\ldots
$$

We form the unique formal inverse

$$
A^{-1}=I-\epsilon R \sum_{|\nu|=3} \alpha_{\nu} \frac{\partial^{|\nu|}}{\partial x^{\nu}}+\ldots
$$

Finally, let $V(x)=A^{-1} h(x)$ and

$$
u(x)=\sum_{\nu} \epsilon^{\nu} \psi_{\nu}(y) \frac{\partial^{|\nu|}}{\partial x^{\nu}} V(x) .
$$

Note that all the infinite series appearing here and in the following only have finitely many non-zero terms. The convergence is trivially true. Then by the claim (1.4),

$$
\begin{aligned}
L u(x) & =\left(L_{0}+\epsilon L_{1}+\epsilon^{2} L_{2}\right) \sum_{|\nu|=0}^{\infty} \epsilon^{|\nu|} \psi_{\nu}(y) \frac{\partial^{|\nu|}}{\partial x^{\nu}} V(x) \\
& =\left(\epsilon^{2} \sum_{|\nu|=2} \alpha_{\nu} \frac{\partial^{|\nu|}}{\partial x^{\nu}}+\epsilon^{3} \sum_{|\nu|=3} \alpha_{\nu} \frac{\partial^{|\nu|}}{\partial x^{\nu}}+\ldots\right) V(x) \\
& =\epsilon^{2} \sum_{|\nu|=2} \alpha_{\nu} \frac{\partial^{|\nu|}}{\partial x^{\nu}} A\left(A^{-1} h(x)\right) \\
& =\epsilon^{2} Q h(x) \\
& =0 .
\end{aligned}
$$

Since $\psi_{0}(y)=1$, one easily verifies that $u^{(N)}(x)=h(x)$. Thus the solution $u(x)$ exists and the proof is complete. 


\section{REFERENCES}

[ALn] M. Avellaneda and F. H. Lin, Une thèorème de Liouville pour des èquations elliptique à coefficients pèriodiques, compt. Rendus Acad. Sci. Paris 309 (1989), 245-250. MR 90j:35072

[KS1] N. V. Krylov and M. V. Safonov, An estimate of the probability that a diffusion process hits a set of positive measure, Soviet Math. Dokl. 20 (1979), 253-255. MR 80b:60101

[KS2] N. V. Krylov and M. V. Safonov, Certain properties of solutions of parabolic equations with measurable coefficients, Izvestia Akad. Nauk. SSSR 40 (1980), 161-175. MR 83c:35059]

[KP] P. Kuchment and Y. Pinchover, Integral representations and Liouville theorems for solutions of periodic equations, preprint.

[L1] P. Li, Harmonic sections of polynomial growth, Math. Research Letters 4 (1997), 35-44. MR 98i:53054

[L2] P. Li, Curvature and function theory on Riemannian manifolds, Survey in Differential Geometry (to appear).

[LW] P. Li and J. Wang, Counting dimensions of L-harmonic functions, Ann. Math. 152 (2000), 645-658.

[Ln] F. H. Lin, Asymptotically conic elliptic operators and Liouville type theorems, Geometric analysis and the calculus of variations, Internat. Press, 1996, pp. 217-238. MR 2000c:35051

[MS] J. Moser and M. Struwe, On a Liouville-type theorem for linear and nonlinear elliptic differential equations on a torus, Bol. Soc. Brasil Mat. 23 (1992), 1-20. MR 94b:35051

[Z] L. Zhang, On the generic eigenvalue flow of a family of metrics and its application, Comm. Anal. Geom. 7 (1999), 259-278. MR 2000f:53053

Department of Mathematics, University of California, Irvine, California 92697-3875

E-mail address: pli@math.uci.edu

School of Mathematics, University of Minnesota, Minneapolis, Minnesota 55455

E-mail address: jiaping@math.umn.edu 